\title{
BMJ Open Systematic review protocol for facilitators and barriers to integrating health services for traumatic brain injury and mental health or addictions
}

Vincy Chan, ${ }^{1}$ Danielle Toccalino, ${ }^{2}$ Angela Colantonio ${ }^{1,2,3}$

To cite: Chan V, Toccalino D, Colantonio A. Systematic review protocol for facilitators and barriers to integrating health services for traumatic brain injury and mental health or addictions. BMJ Open 2019;9:e027534. doi:10.1136/ bmjopen-2018-027534

- Prepublication history and additional material for this paper are available online. To view these files, please visit the journal online (http://dx.doi org/10.1136/bmjopen-2018027534).

Received 26 0ctober 2018 Revised 11 June 2019 Accepted 25 June 2019

Check for updates

(c) Author(s) (or their employer(s)) 2019. Re-use permitted under CC BY-NC. No commercial re-use. See rights and permissions. Published by BMJ.

${ }^{1}$ KITE-Toronto Rehab, University Health Network, Toronto,

Ontario, Canada

${ }^{2}$ Occupational Science \& Occupational Therapy, University of Toronto, Toronto, Ontario,

Canada

${ }^{3}$ Rehabilitation Sciences Institute, University of Toronto, Toronto, Ontario, Canada

Correspondence to

Dr Vincy Chan;

vincy.chan@uhn.ca

\section{ABSTRACT}

Introduction In the most populous province of Canada, one in five adults and one in six students report a lifetime history of traumatic brain injury (TBI). These individuals were also more likely to report elevated psychological distress and use illicit substances compared with those without TBI. The need for integrated health services has been recognised globally, yet efforts to develop more comprehensive and effective care for TBI and mental health and/or addictions (MHA) continue to be challenged by the siloing of the two systems. This protocol is for a systematic review that describes the current types of integrated care for TBI and MHA and identifies the barriers and facilitators to integrating healthcare for these populations.

Methods and analysis This review will systematically search MEDLINE, EMBASE, PsycINF0, CINAHL, Cochrane Central Register of Controlled Trials, Sociological Abstracts, and Dissertations \& Theses Global. References of eligible articles will also be searched for additional relevant studies. The search strategy will include the use of text words and subject headings relevant to the concepts 'TBI,' 'substance abuse, gambling, or mental health,' 'integrated healthcare,' 'barriers and facilitators,' and 'healthcare access.' Two reviewers will independently screen all articles based on predetermined inclusion and exclusion criteria and perform quality assessment on eligible studies. A narrative synthesis will be conducted using the data abstracted by the two reviewers.

Ethics and dissemination Findings from the systematic review will be published in peer-reviewed journals, presented at scientific meetings, and summarised for key stakeholders in the field of TBI and/or MHA. This protocol will form a systematic review that holds the potential to impact policy and planning in the development of integrated person-centred care for TBI and MHA and addresses a recognised gap in TBI care.

Trial registration number CRD42018108343

\section{INTRODUCTION}

Traumatic brain injury (TBI) has been defined as 'an alteration in brain function, or other evidence of brain pathology, caused by an external force. ${ }^{1}$ It is more common than breast cancer, HIV/AIDS, spinal cord injury,

\section{Strengths and limitations of this study}

- The exclusion of non-English language studies, thereby omitting evidence on integrating traumatic brain injury (TBI) and mental health and/or addictions (MHA) in jurisdictions where findings are not published in English, limits the comprehensiveness of the systematic review.

- Recognising not all studies will explicitly describe a policy, intervention, service delivery, or programme as integrating TBI and MHA care, the primary search strategy (TBI, MHA, and integrated care) will be supplemented with an additional search that aims to capture articles describing barriers and facilitators to healthcare for TBI and MHA (TBI, MHA, barriers and facilitators, and healthcare access) to maximise the return of relevant articles.

- At both screening stages, a random selection of articles will first be selected for independent review to enable the reviewers to meet and to establish and document a common extraction and interpretation technique; the reviewers will also meet regularly to review the articles that are included/excluded to ensure consistency in the interpretation of the predetermined inclusion criteria.

and multiple sclerosis combined, ${ }^{2-5}$ and the indirect economic costs due to a TBI is projected to be $\$ 8.2$ billion by 2031 in Canada alone. This exceeds costs of other common neurological conditions, including epilepsy, multiple sclerosis, and dementias including Alzheimer's disease. ${ }^{6}$ In Ontario, Canada, home to $39 \%$ of Canadians, ${ }^{7}$ one in six adults $^{8}$ and one in five students ${ }^{9}$ report a lifetime history of TBI. These individuals were also at least $52 \%$ more likely to have elevated psychological distress, ${ }^{10} 100 \%$ more likely to use cannabis, ${ }^{11} 93 \%$ more likely to experience suicidal ideation, ${ }^{10}$ and $239 \%$ more likely to have attempted suicide ${ }^{10}$ compared with those without a history of TBI. Literature exploring the relationship between TBI and mental health and/or addictions (MHA) 
has found that MHA is prevalent after TBI across the lifespan, particularly diagnoses of schizophrenia, depression, anxiety, substance use disorder, post-traumatic stress disorder, obsessive-compulsive disorder (OCD), and attention deficit hyperactivity disorder (ADHD). ${ }^{12-22}$ It is noteworthy that among individuals with an MHA, a history of TBI is also common. ${ }^{12} 23$

Despite these alarming statistics and the plethora of research establishing a relationship between TBI and MHA, a systems analysis of community and health services for TBI in Ontario identified a lack of appropriate services and community support for individuals with a TBI and MHA. ${ }^{24}$ Efforts to develop more effective and early intervention and care continue to be challenged by the current siloing of the TBI and MHA systems. This need for integrated healthcare has been recognised globally ${ }^{25}{ }^{26}$ and across many health conditions and settings. ${ }^{27-32}$ Most recently, the World Health Assembly adopted the 'Framework on Integrated People-Centred Health Services ${ }^{33}$ with the vision that 'all people have equal access to quality health services that are co-produced in a way that meets their life course needs and respects social preferences, are coordinated across the continuum of care, and are comprehensive, safe, effective, timely, efficient, and acceptable; and all carers are motivated, skilled and operate in a supportive environment. ${ }^{, 33}$ Such integrated care is attractive, as it holds the potential to enable health systems to provide care that addresses the priorities and needs of persons with lived experience, improving their experiences in transitions across their continuum of care, to positively impact patient outcome and quality of life.

Building on this vision, this protocol is for a systematic review that (1) describes the current types of integrated care for TBI and MHA, including access to MHA services for the TBI population and access to TBI services for the MHA population, and (2) identifies the barriers and facilitators to integrating health services for individuals with TBI and MHA. It is recognised that there are numerous concepts and definitions of integration that are currently applied in the literature,$^{33} 34$ as many factors impact the delivery of health services in any given setting, ${ }^{3435}$ making it difficult to identify one definition of health service integration. As such, this protocol will form a systematic review that aims to identify all forms of integration of care (e.g., vertical, horizontal, clinical, and functional integration) to inform policy and programmes to develop integrated care for TBI and MHA.

\section{METHODS AND ANALYSIS \\ Search strategy}

The following databases will be searched for relevant articles:

1. MEDLINE In-Process and Other Non-Indexed Citations and MEDLINE.

2. EMBASE.

3. PsycINFO.

4. CINAHL.
5. Cochrane Central Register of Controlled Trials.

6. Sociological Abstracts.

7. Dissertations \& Theses Global.

The search strategy for this protocol is available through the online supplementary file. This search strategy will include the use of text words and subject headings (e.g., Medical Subject Headings (MeSH), Emtree) related to the following concepts:

A. TBI.

B. Substance abuse, gambling, or mental health.

C. Integrated healthcare.

D. Barrier and facilitator.

E. Healthcare access.

The primary search strategy (concepts $\mathrm{A}+\mathrm{B}+\mathrm{C}$ ) will identify papers that discuss integrated healthcare in a population with TBI and MHA. To complement this primary search strategy, an additional search (concepts $\mathrm{A}+\mathrm{B}+\mathrm{D}+\mathrm{E}$ ) will be included, based on the search strategies of other published reviews ${ }^{27} 3132$ 36-38 and the 'Framework on Integrated People-Centred Health Services,' adopted by the WHO in 2016. ${ }^{33}$ These two searches will be combined with an 'OR' statement to complete the strategy for each of the databases searched, with the exception of the database Sociological Abstracts, where the strategy will be reduced (concepts $\mathrm{A}+\mathrm{B}$ ) due to paucity of results.

Searches will be limited to English language publications and exclude animal studies when possible. Additional limits and search fields will be applied, when applicable, to exclude conference abstracts, magazines, books, and encyclopaedias. Records returned from this search strategy will be managed in EndNote.

\section{Study selection}

For all databases, a first screen will be conducted by two reviewers who will independently assess all titles and abstracts for eligibility based on pre-inclusion criteria:

1. Describe or evaluate a (a) policy, (b) programme, or (c) intervention/treatment at the health service delivery level for individuals with TBI and/or MHA identified in the literature to be prevalent among the TBI population (addictions/substance use, problem gambling, mood and personality disorders, schizophrenia and psychosis, anxiety and depression, trauma and stress disorder, OCD, ADHD, conduct disorder $)^{12-23} ; \underline{\mathrm{OR}}$

2. Screen or diagnosis for a TBI in a health service for MHA; $\underline{\text { OR }}$

3. Screen or diagnosis for a MHA in a health service for TBI; $\underline{\text { AND }}$

4. Quantitative, qualitative, or mixed method studies, or review papers that report primary research findings related to TBI and/or MHA care; $\underline{\text { AND }}$

5. Full-text available.

Articles that meet all of the above criteria will be included for the second screen, which will be a full-text screen. However, articles that are narrative, commentaries, or describe a theory or framework, and articles that look at the broader brain injury population (eg, acquired 
brain injury, patients with trauma) without specific mention of TBI will be excluded.

Recognising that reviewers may interpret the above pre-determined inclusion criteria differently, a random selection of abstracts and titles will first be selected for independent review so the reviewers can meet to establish and document a common extraction and interpretation technique. The reviewers will then meet regularly to review the abstracts that are included/excluded to ensure consistency in the interpretation of the predetermined inclusion criteria.

Two reviewers will independently assess the full-text articles for fulfilment of the following predetermined inclusion criteria:

1. Describe or evaluate a (a) policy, (b) programme, or (c) intervention/treatment at the health service delivery level for individuals with TBI and MHA of interest; $\underline{\mathrm{OR}}$

2. Screen or diagnosis for a TBI in a health service for MHA; $\underline{O R}$

3. Screen or diagnosis for an MHA in a health service for TBI; AND

4. Describe or evaluate an actual, applied experience of integration.

The reference lists of included full-text articles will also be hand-searched for additional relevant articles. The study selection process and the reasons for exclusions at the fulltext level will be presented using the Preferred Reporting Items for Systematic Reviews and Meta-Analyses study flow diagram. Similar to the title and abstract screen, the reviewers will first review a random selection of articles to establish a common extraction and interpretation technique and will meet regularly to ensure consistency in the interpretation of the inclusion criteria.

\section{Data extraction}

The following data will be abstracted independently by two reviewers, with the goal of extracting standardised information that describes the context of the integration activity and associated barriers and facilitators:

1. Author and publication year.

2. Country of study.

3. Funding source (potential conflict of interest from funding? $\mathrm{Y} / \mathrm{N})$.

4. Research objective(s).

5. Study design.

6. Overall and sex-specific/gender-specific participant characteristics and sample size-for example, sociodemographics (age, race/ethnicity, and other measures of socioeconomic status), TBI $(\mathrm{Y} / \mathrm{N}$, severity, cause of injury, time since injury), MHA ( $\mathrm{Y} / \mathrm{N}$ - specify condition(s), time since diagnosis), comorbidities.

7. Description of integration activity.

8. Results of integration activity (stratified by sex/gender, if available).

9. Barriers (stratified by sex/gender, if available).

10. Facilitators (stratified by sex/gender, if available).

11. Additional comments/notes from reviewers.
Similar to the study selection process, the reviewers will first conduct data extraction on select articles to establish a common interpretation and extraction technique and will meet regularly to ensure consistency in the data extracted.

\section{Quality assessment}

Controlled intervention; observational cohort; cross-sectional, case-control, before-after (pre-post) with no control group; and case series studies, as well as systematic reviews and meta-analyses, will be assessed using quality assessment tools created specifically for each study design by the methodologists from the National Institutes of Health and Research Triangle Institute International. ${ }^{39}$ Qualitative studies will be assessed using the Critical Appraisal Skills Programme (CASP) checklist. ${ }^{40}$ Two reviewers will independently assess each article that passes the full-text screen. In the case of rating discrepancies, reviewers will either come to a consensus or a third, independent reviewer will evaluate the study and resolve any disagreements.

\section{Data synthesis}

A narrative synthesis will be conducted using tools and techniques informed by the Guidance on the Conduct of Narrative Synthesis in Systematic Reviews. ${ }^{41}$ The extracted data elements described above and results from the quality assessment will be 'tabulated.' Where data are available, the extracted data elements will be tabulated by sex (if the original study examined sex as it relates to the biological attributes associated with "physical and physiological features including chromosomes, gene expression, hormone levels and functions, and reproductive/sexual anatomy') ${ }^{42}$ and gender (if the original study examined and intended to assess 'the constructed roles, behaviours, expressions, and identifies of girls, women, boys, men, and gender diverse people'). ${ }^{42}$ These findings will be 'grouped and clustered' into the five recommended strategies of the 'Framework on Integrated Person-Centred Care ${ }^{, 33}$ and, where applicable, type/context of integrated care/activity. Finally, a 'textual description' will summarise each included study, focusing on the type of integrated care/activity as it relates to the recommended strategies of the Framework, and associated sex-specific/gender-specific barriers and facilitators.

\section{Patient and public involvement}

Patients and the public were not involved in the creation of this systematic review protocol.

However, this work is part of a larger programme of research entitled 'Integrating Brain Injury, Mental Health, and Addiction', funded by the Ontario Ministry of Health and Long-Term Care, that received input from individuals with lived experience and representatives of organisations that serve the TBI, MHA, housing, criminal justice, and violence against women sectors. 


\section{ETHICS AND DISSEMINATION}

Ethics review will not be required because only publicly available, published data will be analysed. Findings from the systematic review will be published in a peer-reviewed journal, presented at scientific meetings, and summarised for stakeholders of the 'Integrating Brain Injury, Mental Health, and Addictions Research Programme, funded by the Ontario Ministry of Health and Long-Term Care. ${ }^{43}$

\section{Strengths and limitations}

It is recognised that a major limitation of this systematic review is the exclusion of non-English language studies. Evidence on integrating TBI and MHA in jurisdictions where English is not a primary language may be missed, limiting the comprehensiveness of this systematic review. Additionally, unpublished results will not be identified in our systematic review, further limiting its comprehensiveness . Recognising the value of integrated activities whose results are not published in peer-reviewed journals, this protocol includes a process to systematically identify reports and theses to maximise the capture of findings related to integrated care for the TBI and MHA populations.

There are numerous strengths of this review, including those that aim to maximise the retrieval and inclusion of relevant data. The primary search strategy for this review will be supplemented with a secondary search, aimed to capture articles that describe integrations of healthcare but do not describe their study as such. This was added, recognising that some non-specific treatments and interventions, such as screening for a TBI among individuals with MHA, may not be described as a form of integration and would be missed in the primary search. This additional search will ensure that non-specific treatments and integrated care are captured in this review. Similarly, the title and abstract screen will be purposely broad to include articles that describe policy, programmes, or interventions/treatments for individuals with TBI and/ or MHA, recognising that many abstracts may focus on describing data primarily for only the TBI or MHA populations. Including these articles for the full-text screen will reduce the risk of missing relevant articles that describe their study predominantly from the perspective of TBI or MHA. Finally, theses will also be searched to capture early work, such as pilots, of integrated care for TBI and MHA that may not be published in peer-reviewed journals.

To the best of our knowledge, this is the first protocol for a systematic review that describes the types of integrated care for TBI and MHA currently implemented and identifies the barriers and facilitators to integrating care for this population. Informed by the internationally adopted Framework on Integrated Person-Centred Health Services, ${ }^{33}$ findings hold the potential to impact policy and planning for integrated care for TBI and MHA and address a recognised gap in TBI care.

Acknowledgements The authors would like to acknowledge the support of Ms. Jessica Babineau, Information Specialist at the Toronto Rehabilitation
Institute-University Health Network, for assistance in developing the search strategy, and Dr. Tatyana Mollayeva for assistance in identifying appropriate quality appraisal tools for this review.

Contributors VC conceptualised the study, formulated the design, and drafted the manuscript. VC and DT collaborated with JB (Information Specialist) to develop the search strategy. DT and AC critically reviewed the manuscript, and all authors read and approved the final manuscript.

Funding This study was conducted as part of the 2017/18 Health System Research Fund Program Awards, 'Integrating Brain Injury, Mental Health, and Addictions', Ministry Grant No. 267.

Competing interests None declared.

Patient consent for publication Not required.

Provenance and peer review Not commissioned; externally peer reviewed.

Open access This is an open access article distributed in accordance with the Creative Commons Attribution Non Commercial (CC BY-NC 4.0) license, which permits others to distribute, remix, adapt, build upon this work non-commercially, and license their derivative works on different terms, provided the original work is properly cited, appropriate credit is given, any changes made indicated, and the use is non-commercial. See: http://creativecommons.org/licenses/by-nc/4.0/.

\section{REFERENCES}

1. Menon DK, Schwab K, Wright DW, et al. Position statement: definition of traumatic brain injury. Arch Phys Med Rehabil 2010;91:1637-40.

2. Challacombe L. The Epidemiology of HIV in Canada. 2018 https:// www.catie.ca/fact-sheets/epidemiology/epidemiology-hiv-canada: CATIE (accessed 11 Sep 2018).

3. Cancer Care Ontario. Ontario Cancer Statistics 2018. Toronto, Ontario: Cancer Care Ontario, 2018:172.

4. Noonan VK, Fingas M, Farry A, et al. Incidence and prevalence of spinal cord injury in Canada: a national perspective. Neuroepidemiology 2012;38:219-26.

5. Gilmour H, Ramage-Morin PL, Wong SL. Multiple sclerosis: Prevalence and impact. Health Reports: Statistics Canada, 2018:3-8.

6. Mapping Connections: An understanding of neurological conditions in Canada. The National Population Health Study of Neurological Conditions. 2014 https://www.canada.ca/en/public-health/services/ reports-publications/mapping-connections-understandingneurological-conditions.html.

7. Statistics Canada. Table 17-10-0005-01 Population estimates on July 1st, by age and sex. 2018 https://www150.statcan.gc.ca/t1/tbl1/en/ cv.action?pid=1710000501 (accessed 11 Sep 2018).

8. Ilie G, Adlaf EM, Mann RE, et al. Associations between a history of traumatic brain injuries and current cigarette smoking, substance use, and elevated psychological distress in a population sample of canadian adults. J Neurotrauma 2015;32:1130-4.

9. Ilie G, Boak A, Adlaf EM, et al. Prevalence and correlates of traumatic brain injuries among adolescents. JAMA 2013;309:2550-2.

10. Ilie G, Mann RE, Boak A, et al. Suicidality, bullying and other conduct and mental health correlates of traumatic brain injury in adolescents. PLoS One 2014;9:e94936.

11. Ilie G, Mann RE, Hamilton $\mathrm{H}$, et al. Substance use and related harms among adolescents with and without traumatic brain injury. $J$ Head Trauma Rehabil 2015;30:293-301.

12. Adeyemo BO, Biederman J, Zafonte R, et al. Mild traumatic brain injury and ADHD: a systematic review of the literature and metaanalysis. J Atten Disord 2014;18:576-84.

13. Whelan-Goodinson R, Ponsford J, Johnston L, et al. Psychiatric disorders following traumatic brain injury: their nature and frequency. J Head Trauma Rehabil 2009;24:324-32.

14. Max JE, Lansing AE, Koele SL, et al. Attention deficit hyperactivity disorder in children and adolescents following traumatic brain injury. Dev Neuropsychol 2004;25(1-2):159-77.

15. Max JE, Wilde EA, Bigler ED, et al. Psychiatric disorders after pediatric traumatic brain injury: a prospective, longitudinal, controlled study. J Neuropsychiatry Clin Neurosci 2012;24:427-36.

16. Orlovska S, Pedersen MS, Benros ME, et al. Head injury as risk factor for psychiatric disorders: a nationwide register-based followup study of 113,906 persons with head injury. Am J Psychiatry 2014;171:463-9.

17. Schachar RJ, Park LS, Dennis M. Mental Health Implications of Traumatic Brain Injury (TBI) in Children and Youth. J Can Acad Child Adolesc Psychiatry 2015;24:100-8. 
18. Bryant RA, O'Donnell ML, Creamer M, et al. The psychiatric sequelae of traumatic injury. Am J Psychiatry 2010;167:312-20.

19. Silver JM, Kramer R, Greenwald S, et al. The association between head injuries and psychiatric disorders: findings from the New Haven NIMH Epidemiologic Catchment Area Study. Brain Inj 2001;15:935-45.

20. Perry DC, Sturm VE, Peterson MJ, et al. Association of traumatic brain injury with subsequent neurological and psychiatric disease: a meta-analysis. J Neurosurg 2016;124:511-26.

21. Massagli TL, Fann JR, Burington BE, et al. Psychiatric illness after mild traumatic brain injury in children 11 No commercial party having a direct financial interest in the results of the research supporting this article has or will confer a benefit on the author(s) or on any organization with which the author(s) is/are associated. Arch Phys Med Rehabil 2004;85:1428-34.

22. Gibson R, Purdy SC. Mental health disorders after traumatic brain injury in a New Zealand caseload. Brain Inj 2015;29:306-12.

23. Albicini M, Eggleston M, McKinlay A. The prevalence of traumatic brain injury, comorbid anxiety and other psychiatric disorders in an outpatient child and adolescent mental health service. J Ment Health 2017:5:1-7.

24. Munce SE, Laan RV, Levy C, et al. Systems analysis of community and health services for acquired brain injury in Ontario, Canada. Brain Inj 2014;28:1042-51.

25. Legido-Quigley $\mathrm{H}$, Montgomery $\mathrm{CM}$, Khan $\mathrm{P}$, et al. Integrating tuberculosis and HIV services in low- and middle-income countries: a systematic review. Trop Med Int Health 2013;18:199-211.

26. Sigfrid L, Murphy G, Haldane V, et al. Integrating cervical cancer with HIV healthcare services: A systematic review. PLoS One 2017; 12:e0181156.

27. Atun R, de Jongh T, Secci F, et al. A systematic review of the evidence on integration of targeted health interventions into health systems. Health Policy Plan 2010;25:1-14.

28. Chuah FLH, Haldane VE, Cervero-Liceras F, et al. Interventions and approaches to integrating HIV and mental health services: a systematic review. Health Policy Plan 2017;32(suppl_4):iv27-iv47.

29. Haldane V, Cervero-Liceras F, Chuah FL, et al. Integrating HIV and substance use services: a systematic review. J Int AIDS Soc 2017;20:21585.
30. Haldane V, Legido-Quigley H, Chuah FLH, et al. Integrating cardiovascular diseases, hypertension, and diabetes with HIV services: a systematic review. AIDS Care 2018;30:103-15

31. Wakida EK, Akena D, Okello ES, et al. Barriers and facilitators to the integration of mental health services into primary health care: a systematic review protocol. Syst Rev 2017;6:171.

32. Watt N, Sigfrid L, Legido-Quigley $\mathrm{H}$, et al. Health systems facilitators and barriers to the integration of HIV and chronic disease services: a systematic review. Health Policy Plan 2017;32(suppl_4):iv13-iv26.

33. Secretariat WHO. Framework on Integrated People-Centred Health Services. Geneva, Switzerland: World Health Organization, 2016.

34. Suter E, Oelke ND, Adair CE, et al. Health systems integration. definitions, processes \& impact: a research synthesis: Alberta Health Services, 2007.

35. Heyeres M, McCalman J, Tsey K, et al. The complexity of health service integration: a review of reviews. Front Public Health 2016;4:223.

36. Balogh R, McMorris CA, Lunsky Y, et al. Organising healthcare services for persons with an intellectual disability. Cochrane Database Syst Rev 2016;4:CD007492.

37. Briggs CJ, Garner P. Strategies for integrating primary health services in middle- and low-income countries at the point of delivery. Cochrane Database Syst Rev 2006;2:CD003318.

38. Kantor V, Knefel M, Lueger-Schuster B. Perceived barriers and facilitators of mental health service utilization in adult trauma survivors: A systematic review. Clin Psychol Rev 2017;52:52-68.

39. National Heart, Lung, and Blood Institute. Background: Development and Use of Study Quality Assessment Tools. https://www.nhlbi.nih. gov/node/80102 (accessed 10 Sep 2018).

40. Programme CAS. CASP Qualitative Checklist. https://casp-uk.net/ wp-content/uploads/2018/01/CASP-Qualitative-Checklist.pdf2018 (accessed 10 Sep 2018)

41. Popay J, Roberts H, Sowden A, et al. Guidance on the conduct of narrative synthesis in systematic reviews. A product from the ESRC methods programme Version. 2006;1:b92.

42. Definitions of Sex and Gender Canada. Canadian Institutes of Health Research. 2015 http://www.cihr-irsc.gc.ca/e/47830.html (accessed 21 Sep 2018).

43. Chan V, Balogh R, Grigorovich A, et al. Integrating brain injury, mental health, and addictions research program. Brain Inj 2019;33(S1):213. 